\title{
Emergency department over-crowding: a global perspective
}

\author{
Latha G. Stead • Anunaya Jain • Wyatt W. Decker
}

Published online: 30 September 2009

(C) Springer-Verlag London Ltd 2009

Emergency department (ED) over-crowding has been a topic of intense interest over the past few years, with the Institute of Medicine report "Emergency Care at a Crossroads" published in 2006 [1], followed by the American College of Emergency Physicians task force report on boarding (2008) and, most recently, the United States Government Accountability Office (GAO) Report to the Chairman [2], Committee on Finance, U.S. Senate published in April 2009. Similarly, the Canadian Association of Emergency Physicians and the Australasian College for Emergency Medicine have also recognized the primary problem of over-crowding in the ED [3, 4].

The adverse effects of the global problem of ED overcrowding are well known. Crowding negatively impacts all stakeholders: patients, physicians, and the hospital. Most important is patient safety, with decreased quality of care and an increase in medical errors in overcrowded EDs. Further, patients have a poor experience, which leads them to leave without being seen, and they are less likely to return to the ED in the future. Physicians and other providers experience decreased job satisfaction, resulting in decreased productivity and increased staff turnover. For the hospital, ED over-crowding results in lost revenue from multiple sources. Revenue is lost from patients who leave without being seen, from emergency medical service (EMS) diversion secondary to dissatisfaction among both patients and EMS crews, and from shifting of the market share to

L. G. Stead $\cdot$ A. Jain $(\bowtie) \cdot$ W. W. Decker

University of Rochester, School of Medicine \& Dentistry, 601 Elmwood Ave., Box 655, Rochester, NY 14620, USA

e-mail: anunaya_jain@urmc.rochester.edu

L. G. Stead · A. Jain $\cdot$ W. W. Decker

University of Rochester Medical Center,

Rochester, NY 14620, USA competitors. Consider the real mortality risk associated with ED over-crowding. A study of over 55,000 ED patients demonstrated a higher mortality rate among patients boarded in the ED than in those moved to an inpatient hallway bed $(p<0.05)$. Furthermore, the Joint Commission on the Accreditation of Healthcare Organizations (JCAHO) notes that $50 \%$ of sentinel events occur in the ED and of these one third are due to over-crowding.

Hospitals and EDs react in a crisis. Institutions, stressed by overcrowding, often engage in short-term fixes which do not address the underlying issues, and ultimately only exacerbate the problem. For example, increased staffing and expansion of the ED do not necessarily produce gains. Rather, redesigning for optimization of the system by which patients are processed is the key. The secret to success is not to allocate more, but to strategically re-allocate resources.

In 2001, a survey of 575 ED directors reported overcrowding in $94 \%$ of academic EDs and $91 \%$ of private hospital EDs [5]. In this time of economic downturn, the issue of ED over-crowding becomes even more poignant as a global issue. This issue of International Journal of Emergency Medicine features two studies that highlight the problem of ED over-crowding.

In their 7-year longitudinal study, Graham et al. [6] report on the trends in Prince of Wales Hospital, a busy $(>155,000$ patient visits per year) tertiary referral facility with 1,400 beds which serves as the primary teaching hospital of the Chinese University of Hong Kong. The authors note that while the SARS epidemic and the introduction of co-pay in the ED resulted in an overall net decrease of ED volumes, the concurrent increase in patients brought in via ambulance plus reduction of medical and nursing staff resulted in increased waiting times. The overcrowding problem is compounded by the marked increase 
in the medical admission rate, which correlates with the $12 \%$ increase in the geriatric population visits.

The second study, by Kulstad and Kelley [7], entitled "Over-crowding is associated with delays in percutaneous coronary intervention for acute myocardial infarction" uses the EDWIN score as a marker of ED over-crowding and its association with ultimate delays in patients receiving percutaneous intervention for acute myocardial infarction. The EDWIN score is the sum of the number of patients in the ED multiplied by their acuity, divided by the number of attending physicians working in the ED, multiplied by the number of inpatient boarders. The authors report a decreased likelihood of timely treatment for acute MI during times of over-crowding in their emergency department.

These studies underscore the very real and detrimental effects of ED over-crowding. The most important root cause of ED over-crowding is the inability to transfer emergency patients to inpatient beds and the resultant boarding of admitted patients in the ED. Solutions to decrease boarding in the ED include moving boarders into inpatient hallways at the front end, and increasing the rate of discharges by noon at the back end. Movement of patients to inpatient hallways, while known to be safer for patients, still often results in push-back from the inpatient services. One commonly cited reason is patient dissatisfaction. As the science of ED overcrowding evolves, studies addressing this very issue have also been performed. A study of 445 patients reports an overall preference of $87 \%$ for inpatient hallway beds. Reasons cited for this preference included better privacy, staff availability, safety, rest, quiet, and treatment [8]. Movement of patients to hallway beds and boosting discharges before noon are sometimes thought of as the "external factor," as they involve practice outside of the ED, namely inpatient floors. There are of course a number of measures that can be undertaken to increase throughput in the ED that can be considered "internal factors." These include streamlined and simplified triage, point-of-care laboratory testing for diagnostics that are a bottleneck to the next step, and care pathways, to name a few.

The first step of solving any problem, acknowledging it, is well past. We know that patient volumes of EDs are going to increase at an exponential rate, as suggested by the CDC in
2005 [9], Our objectives should be to enable efficient care to everyone at all times. We have potential allies in technology, streamlined business management strategies, including Lean for process evolution and Six Sigma, and collaborative alliances between hospitals to develop creative solutions to the problem of ED over-crowding.

In today's evolving healthcare scenario, EDs are not only a point of care for the acutely ill, but also have a role as a safety net to provide healthcare to people regardless of their insurance status or ability to pay, as dictated by laws like the Emergency Medical Treatment and Labor Act (EMTALA) in the US. It is of paramount importance that a serious and unified approach to ED over-crowding be taken, at all levels. This would represent a major step towards establishing a culture of patient safety and satisfaction in the ED.

\section{References}

1. Institute of Medicine (US) Committee on the Future of Emergency Care in the United States Health System (2006) Hospital-based emergency care: at the breaking point. Washington, DC; ISBN-10: 0-309-10173-5

2. US Government Accountability Office (2009) Emergency department crowding. GAO-09-347

3. Romanow Commission: CAEP submission on emergency department overcrowding. http://www.caep.ca/page.asp?id=C66C924CF39543 D9AF7E62BAB1A67835

4. ACEM statement on emergency department overcrowding. http://www. acem.org.au/media/policies_and_guidelines/S57_-_Statement_on ED_Overcr.pdf

5. Derlet R, Richards J, Kravitz R (2001) Frequent overcrowding in U.S. emergency departments. Acad Emerg Med 8(2):151-155

6. Wai AKC, Chor CM, Lee ATC, Sittambunka Y, Graham CA, Rainer TH (2009) Analysis of trends in emergency department attendances, hospital admissions and medical staffing in a Hong Kong university hospital: 5-year study. Int J Emerg Med. doi:10.1007/s12245-009-0107-x

7. Kulstad EB, Kelly KM (2009) Crowding is associated with delays in percutaneous coronary intervention for acute myocardial infarction. Int J Emerg Med. doi:10.1007/s12245-009-0098-7

8. Viccellio A, Santora C, Singer AJ, Thode HC Jr, Henry MC (2009) The association between transfer of emergency department boarders to inpatient hallways and mortality: a 4-year experience. Ann Emerg Med Apr 2 [Epub ahead of print]

9. National Hospital Ambulatory Medical Care Survey: 2005 Emergency Department Summary. http://www.cdc.gov/nchs/data/ad/ ad386.pdf 